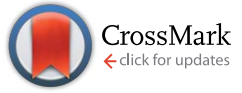

Cite this: RSC Adv., 2016, 6, 115345

DOI: 10.1039/c6ra90130a

www.rsc.org/advances

\title{
Retraction: 3D small molecule microarray with enhanced sensitivity and immobilization capacity monitored by surface plasmon resonance imaging
}

\author{
Jinsong Zhu ${ }^{\mathrm{ab}}$ \\ Retraction of '3D small molecule microarray with enhanced sensitivity and immobilization capacity \\ monitored by surface plasmon resonance imaging' by Vikramjeet Singh et al., RSC Adv., 2014, DOI: \\ 10.1039/c4ra07306a.
}

I, Jingsong Zhu, the corresponding author, hereby wholly retract this RSC Advances article.

After the publication of the article it has been identified that there are a number of flaws in the results presented. Further repeats of the reported kinetics experiments have identified that there is in fact no difference between the $2 \mathrm{D}$ and $3 \mathrm{D}$ surfaces. In addition, the authors did not take account of the effect of non-specific binding on the $K_{\mathrm{D}}$ values reported. Finally, recent characterisation experiments have confirmed that the samples of inhibitors SB239063 and SB202190 used for this study were in fact the same compound. This was overlooked initially due to errors in the characterisation. Therefore, the corresponding author no longer has confidence that the conclusions of this RSC Advances paper can be adequately supported.

The corresponding author regrets these oversights and apologises for any inconvenience to authors and readers.

Signed: Jinsong Zhu

8th November 2016.

Vikramjeet Singh and Amita Nand oppose this retraction and support the accuracy of the results presented. Zhiqiang Cheng and Mo Yang could not be contacted.

Retraction endorsed by Andrew Shore, Executive Editor, RSC Advances. 